\title{
Estresse e garantia do direito à saúde de policiais militares: uma revisão sistemática
}

\author{
Stress and guarantee of the right to health of military police: a systematic review \\ Estrés y garantía del derecho a la salud de la policía militar: una revisión sistemática
}

Recebido: 09/10/2021 | Revisado: 19/10/2021 | Aceito: 20/10/2021 | Publicado: 22/10/2021

Werick Medeiros Alves
ORCID: https://orcid.org/0000-0001-9284-5139
Academia de Polícia Militar de Alagoas Senador Arnon de Mello, Brasil
E-mail: werickmedeiros@ hotmail.com
Verônica de Medeiros Alves
ORCID: https://orcid.org/0000-0002-4343-2941
Universidade Federal de Alagoas, Brasil
E-mail: veronica.alves@eenf.ufal.br
Priscilla Souza dos Santos
ORCID: https://orcid.org/0000-0002-8876-5352
Universidade Federal de Alagoas, Brasil
E-mail: prys.dossantos@gmail.com
Wellington da Silva Lima Santos
ORCID: https://orcid.org/0000-0002-3953-5964
Centro Universitário CESMAC, Brasil
E-mail: wellington.limasantos@ hotmail.com
Elton Lima Santos
ORCID: https://orcid.org/0000-0002-0965-5332
Universidade Federal de Alagoas, Brasil
E-mail: Elton.santos@ceca.ufal.br
Ellen Vidal Medeiros Lobo
ORCID: https://orcid.org/0000-0002-3625-0605
Universidade Federal de Alagoas, Brasil
E-mail: vidalenf@gmail.com
Ana Paula Nogueira de Magalhães
ORCID: https://orcid.org/0000-0002-5071-0778
Universidade Federal de Alagoas, Brasil
E-mail: paula_nog@arapiraca.ufal.br

\section{Resumo}

A compreensão do estresse eleva as condições para que se possa melhorar a qualidade de vida dos policiais militares. Sendo assim, este trabalho tem como objetivo identificar como se apresenta o estresse em policiais militares, descritos nas produções científicas. Trata-se de uma revisão sistemática da literatura, onde se adotou os critérios do Preferred Reporting Items for Systematic Reviews and Meta-Analyses (PRISMA). A estratégia de busca adotada foi uma investigação dos artigos nas bases de dados eletrônicas: Lilacs, Scielo e Medline/PubMed. Os termos utilizados na pesquisa foram baseados nos descritores em Ciências da Saúde (DECs), considerando-se as seguintes palavras: “military police” OR “policía militar” OR "Polícia Militar” AND “psychological stress” OR "Estrés Psicológico” OR "Estresse Psicológico". Os critérios de inclusão adotados nesta pesquisa foram: o artigo deveria estar indexado nas bases de dados selecionadas e disponível em inglês, espanhol ou português na íntegra; com participantes da pesquisa de qualquer sexo e idade; pesquisas quantitativas e/ou qualitativas. Observou-se que a interação interpessoal baseada em características de hostilidade, problemas com a hierarquia, grande demanda de trabalho e preconceito de gênero foram identificados como fatores geradores de estresse. Os estudos mostram que os policiais apresentaram estresse com sintomas psicológicos e sintomas físicos. Nesse contexto, sugere-se que a elaboração de ações de promoção de saúde e prevenção dos transtornos mentais em policiais militares são necessárias visando melhorar as condições laborais geradoras de estresse.

Palavras-chave: Estresse; Polícia militar; Trabalho.

\begin{abstract}
Understanding stress raises the conditions for improving the quality of life of military police officers. Thus, this work aims to identify how stress is presented in military police, described in scientific productions. This is a systematic literature review, where the Preferred Reporting Items for Systematic Reviews and Meta-Analyses (PRISMA) criteria were adopted. The search strategy adopted was an investigation of articles in electronic databases: Lilacs, Scielo and Medline/PubMed. The terms used in the research were based on Health Sciences descriptors (DECs), considering the following words: "military police" OR "policía militar" OR “polícia militar" AND "psychological stress" OR "Estrés Psicológico" OR "Estresse Psicológico". The inclusion criteria adopted in this research were: the article should be
\end{abstract}


indexed in the selected databases and available in full English, Spanish or Portuguese; with survey participants of any gender and age; quantitative and/or qualitative research. It was observed that interpersonal interaction based on hostility characteristics, problems with the hierarchy, high work demand and gender bias were identified stress generators. Studies show that the police presented stress with psychological symptoms and physical symptoms. In this context, it is suggested that the elaboration of actions to promote health and prevent mental disorders in military police officers are necessary in order to improve the working conditions that generate stress.

Keywords: Stress; Military police; Work.

\section{Resumen}

Comprender el estrés plantea las condiciones para mejorar la calidad de vida de los agentes de la policía militar. Así, este trabajo tiene como objetivo identificar cómo se presenta el estrés en la policía militar, descrito en producciones científicas. Se trata de una revisión sistemática de la literatura, en la que se adoptaron los criterios de elementos de informe preferidos para revisiones sistemáticas y metaanálisis (PRISMA). La estrategia de búsqueda adoptada fue una investigación de artículos en bases de datos electrónicas: Lilacs, Scielo y Medline / PubMed. Los términos utilizados en la investigación se basaron en descriptores de Ciencias de la Salud (DECs), considerando las siguientes palabras: "military police" O "policía militar" O "polícia militar" Y "psychological stress O "estrés psicológico" O "estresse psicológico". Los criterios de inclusión adoptados en esta investigación fueron: el artículo debe estar indexado en las bases de datos seleccionadas y estar disponible en su totalidad en inglés, español o portugués; con participantes de la encuesta de cualquier género y edad; investigación cuantitativa y / o cualitativa. Se observó que la interacción interpersonal basada en características de hostilidad, problemas con la jerarquía, alta demanda laboral y sesgo de género fueron identificados como generadores de estrés. Los estudios demuestran que la policía presentó estrés con síntomas psicológicos y físicos. En este contexto, se sugiere que la elaboración de acciones para promover la salud y prevenir los trastornos mentales en los oficiales de la policía militar son necesarias para mejorar las condiciones de trabajo que generan estrés.

Palabras clave: Estrés; Policía militar; Trabaja.

\section{Introdução}

Os policiais militares são indivíduos que, em caráter permanente ou transitório, prestam serviços militares no plano da administração da União e dos Estados. Sendo assim, os policiais militares se referem aos profissionais que desempenham atividade no âmbito federal ou no estadual, recebendo por este serviço um subsídio (Bassalo et al., 2020).

As atividades laborais do policiamento ostensivo mostram um pouco das relações entre o ambiente de trabalho policial militar e sua qualidade de vida. A avaliação da presença de estresse no trabalho pode auxiliar os Comandantes de Unidades da Polícia Militar na tomada de decisões referente aos anseios do público interno a respeito do ambiente de trabalho mais saudável, a fim de solucionar de forma eficaz e baseada em evidências científica, problemas que possam estar afetando o ambiente laboral na prestação de serviços de qualidade (Dorileo \& Souza, 2017).

O estresse é um mecanismo que compromete diretamente a saúde mental. Nesta situação, o organismo não corresponde a uma resposta específica equilibrada, podendo assim, esta ser positiva ou negativa. Os eventos estressores negativos são situações que ameaçam a saúde e podem identificá-lo pelo nível de ansiedade acarretada pelo indivíduo. Evidencia-se então, que quanto mais crônico se encontrar o estado de ansiedade, maiores as consequências geradas para o organismo por essa situação de estresse (Farné, 2003).

O estresse pode ser classificado em quatro fases: a) Alerta, em que o ser humano através da produção da adrenalina energiza-se a fim de buscar a sobrevivência e a sensação de plenitude é alcançada. b) Resistência, a pessoa busca lidar com seus estressores de modo a manter sua homeostase interna. Se os fatores estressantes persistirem em frequência ou intensidade e, se nesta fase, ocorrer uma quebra na resistência da pessoa, ela passará para próxima fase. c) Quase-exaustão, fase em que a pessoa se encontra em um processo de adoecimento onde os órgãos que tiverem uma maior vulnerabilidade genética ou adquirida passa a mostrar sinais de deterioração. Se não minimizar o estresse atinge a sua fase final. d) Exaustão, quando doenças graves podem acontecer nos órgãos mais vulneráveis (Lipp, 2005).

Segundo a Constituição Federal, a saúde é um direito fundamental. No artigo 196, consta que a saúde é direito de todos e dever do Estado, garantindo que todos têm direito a tratamentos adequados, fornecidos pelo poder público (Brasil, 
1988). Partindo dessa perspectiva, é importante refletir que os policiais, cuidando da segurança coletiva, são também sujeitos de direito, servidores públicos protegidos pela Constituição, que lhes assegura integridade física e mental no desempenho de suas atividades (Souza \& Minayo, 2005).

Dessa forma, faz-se necessário para a atuação da polícia, uma assistência psicossocial, tendo em vista que estes profissionais de regime rígido devem transmitir uma sensação de paz, tranquilidade, transparência e solidariedade entre a população de sua área de atuação; fazendo suas ações voltadas para o bem comum e para o trabalho com qualidade profissional. Nesse contexto, levanta-se a problemática: como se apresenta o estresse em policiais militares, descritos nas produções científicas?

Devido ao fato de que os policiais militares lidarem rotineiramente com situações estressantes e que exigem equilíbrio e conhecimento prévio, conhecer como se apresenta os níveis de estresse se faz necessário para poder contribuir com estratégias que possibilitem tomadas de decisão mais assertivas e previnam o adoecimento do mesmo.

É pertinente identificar como se apresenta o estresse em policiais militares, descritos nas produções científicas, levando em consideração a qualidade da saúde mental como um direito fundamental.

\section{Metodologia}

Trata-se de uma revisão sistemática da literatura, onde se adotou os critérios do Preferred Reporting Items for Systematic Reviews and Meta-Analyses (PRISMA). O PRISMA orienta a formulação e a redação de uma revisão sistemática e resume informações relativas ao objetivo do pesquisador (Liberati et al., 2009; Shamseer et al., 2015).

A formulação da pergunta que serviu de base para a pesquisa e seu título foi fundamentada no método "Participants, Interventions, Comparators, Outcomes, and Study design" (PICOS) (Wright et al., 2016), que serviu para subsidiar informações precisas sobre o domínio da pergunta disparadora empregada para realizar a pesquisa. PICOS: Participantes: Policiais militares. Intervenção: Estresse. Comparação: Não tem. Resultados: Fatores geradores de estresse. Tipo de estudo: Transversal.

A estratégia de busca adotada foi a investigação dos artigos nas bases de dados eletrônicas: Lilacs, Scielo e Medline/PubMed. Os termos utilizados na pesquisa foram baseados nos descritores em Ciências da Saúde (DECs), considerando-se as seguintes palavras: "military police" OR "policía militar" OR "Polícia Militar" AND "psychological stress" OR "Estrés Psicológico" OR "Estresse Psicológico".

Os critérios de inclusão adotados nesta pesquisa foram: o artigo deveria estar indexado nas bases de dados selecionadas e disponível em inglês, espanhol ou português na íntegra; com participantes da pesquisa de qualquer sexo e idade; pesquisas quantitativas e/ou qualitativas.

Os artigos foram selecionados hierarquicamente pelos seguintes elementos: título; duplicação; resumo; disponibilidade completa de forma livre do texto; leitura do texto e respeito aos critérios de inclusão. A coleta dos dados foi iniciada no dia 22 de maio de 2020, e concluída em 29 de maio do mesmo ano. Uma primeira análise se deu com base nas informações obtidas no título e no resumo dos artigos encontrados e, num segundo momento, os estudos foram lidos e avaliados na íntegra.

\section{Resultados e Discussão}

Foram identificados 244 estudos potencialmente elegíveis para essa revisão, dos quais 21 foram encontrados na LILACS, 18 na Scielo e 205 no Medline/Pubmed (Figura 1). 
Figura 1. Fluxograma dos artigos selecionados.

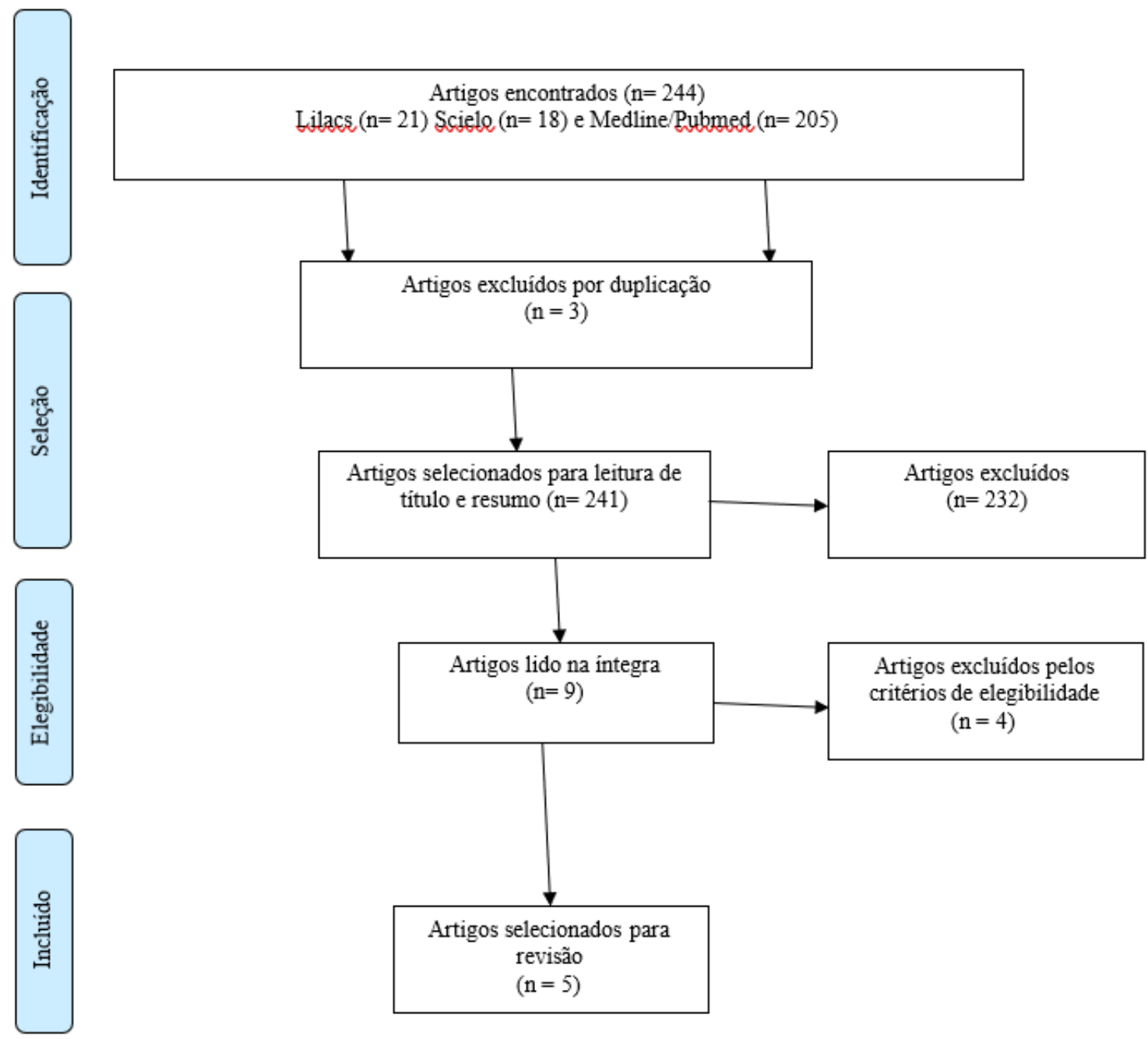

Fonte: Autores.

Três artigos foram descartados por serem duplicados e 232 foram excluídos depois de lidos os títulos e resumos, restando nove (9) artigos para leitura na íntegra. Quatro artigos foram excluídos mediante os critérios de elegibilidade e inclusão descritos anteriormente. Sendo assim, restaram 05 artigos para a síntese qualitativa que atenderam os critérios de inclusão pré-determinados. Estes artigos estão apresentados no quadro 1, onde é possível identificar: autor, ano de publicação, amostra do estudo, abordagem do estudo, local, fatores geradores de estresse e conclusão. Os estudos selecionados foram publicados nos anos de 2007, 2008, 2012, 2013 e 2017 (Costa et al., 2007; Carvalho et al., 2008; Couto, Vandenberghe \& Brito, 2012; Bezerra, Minayo \& Constantino, 2013; Tavares et al., 2017).

A amostra de policiais nos estudos variava muito, pois, foram encontrados estudos com 3.193 militares do Comando de Policiamento da Capital (Costa et al., 2007), 327 alunos dos cursos de Formação de Oficiais da Polícia Militar (Couto, Vandenberghe \& Brito, 2012), 134 policiais militares do Batalhão de Operações Especiais (Tavares et al., 2017), 81 policiais do sexo masculino (Carvalho et al., 2008) e 42 mulheres oficiais e praças, profissionais operacionais e de saúde (Bezerra, Minayo \& Constantino, 2013). Dentre a amostra vale ressaltar ainda que quatro estudos eram quantitativos e transversais (Costa et al., 2007; Carvalho et al., 2008; Couto, Vandenberghe \& Brito, 2012; Tavares et al., 2017) e um era qualitativo (Bezerra, Minayo \& Constantino, 2013). 
Research, Society and Development, v. 10, n. 13, e592101321597, 2021

(CC BY 4.0) | ISSN 2525-3409 | DOI: http://dx.doi.org/10.33448/rsd-v10i13.21597

Quadro 1. Dados coletados dos artigos selecionados para a revisão sistemática.

\begin{tabular}{|c|c|c|c|c|c|}
\hline Autor (Ano) & Amostra & Abordagem & Local & Fatores de estresse & Conclusão \\
\hline Carvalho et al., 2008 & $\begin{array}{l}81 \text { policiais do sexo } \\
\text { masculino }\end{array}$ & $\begin{array}{l}\text { Estudo transversal. } \\
\text { Os voluntários foram submetidos à } \\
\text { anamnese exame clínico. } \\
\text { Para o diagnóstico de estresse foi } \\
\text { aplicado o Inventário de Sintomas do } \\
\text { Stress (Stress Symptoms Inventory - } \\
\text { SSI). }\end{array}$ & São Luís, MA & $\begin{array}{l}26,7 \% \text { dos policiais da administração e } 10,6 \% \text { do } \\
\text { grupo com atividade operacional apresentaram } \\
\text { estresse. entre os policiais bruxistas, 33,3\% } \\
\text { relataram desconforto nos músculos } \\
\text { mastigatórios, enquanto } 25,9 \% \text { apresentaram } \\
\text { sensibilidade à palpação dos músculos temporal } \\
\text { e/ou masseter. }\end{array}$ & $\begin{array}{l}\text { A prevalência de bruxismo foi de } 33,3 \% \text { e a de } \\
\text { estresse emocional, foi de } 13,6 \% \text {. } \\
\text { A prevalência de estresse em indivíduos com } \\
\text { bruxismo foi significativamente maior em } \\
\text { relação aos indivíduos sem bruxismo (teste } \\
\text { qui-quadrado, } \mathrm{P}=0,003 \text { ). }\end{array}$ \\
\hline Costa et al., 2007 & $\begin{array}{l}3.193 \text { militares do } \\
\text { Comando de } \\
\text { Policiamento da } \\
\text { Capital }\end{array}$ & $\begin{array}{l}\text { Inventário de Sintomas de Stress para } \\
\text { Adultos de Lipp. }\end{array}$ & Natal, Brasil & $\begin{array}{l}\text { Associação positiva entre sexo feminino e } \\
\text { estresse }(p=0,0337) \text {. }\end{array}$ & $\begin{array}{l}\text { Dos } 264 \text { policiais investigados, } 125(47,4 \%) \\
\text { apresentaram estresse. } \\
\text { Destes, } 3,4 \% \text { encontravam-se na fase de alerta, } \\
39,8 \% \text { na fase de resistência, } 3,8 \% \text { na fase de } \\
\text { quase-exaustão e } 0,4 \% \text { na fase de exaustão. } \\
\text { Sintomas psicológicos foram registrados em } \\
76,0 \% \text { dos policiais com estresse, e sintomas } \\
\text { físicos em } 24,0 \% \text {. }\end{array}$ \\
\hline $\begin{array}{c}\text { Bezerra, Minayo \& } \\
\text { Constantino, } 2013\end{array}$ & $\begin{array}{l}42 \text { mulheres: oficiais } \\
\text { e praças, } \\
\text { profissionais } \\
\text { operacionais e de } \\
\text { saúde }\end{array}$ & $\begin{array}{l}\text { Abordagem qualitativa (entrevistas, } \\
\text { grupos focais e observação) }\end{array}$ & Rio de Janeiro & $\begin{array}{l}\text { Fatores de estresse negativo as policiais } \\
\text { destacam: problemas com a hierarquia, grande } \\
\text { demanda de trabalho e preconceito de gênero. } \\
\text { O sofrimento psíquico aparece mais entre as } \\
\text { oficiais com cargos de chefia; e as atividades } \\
\text { operacionais são percebidas como mais } \\
\text { estressantes pelo risco que oferecem. }\end{array}$ & $\begin{array}{l}\text { O exercício físico é a estratégia considerada } \\
\text { mais eficaz para prevenir as consequências do } \\
\text { estresse. } \\
\text { A organização e o gerenciamento continuam } \\
\text { sob a ótica masculina, sendo necessário } \\
\text { investimentos em ações preventivas do } \\
\text { estresse sob a perspectiva de gênero. }\end{array}$ \\
\hline $\begin{array}{l}\text { Couto, Vandenberghe, } \\
\text { Brito, } 2012\end{array}$ & $\begin{array}{l}327 \text { alunos dos } \\
\text { cursos de Formação } \\
\text { de Oficiais da } \\
\text { Polícia Militar }\end{array}$ & $\begin{array}{l}\text { Inventário de Sintomas de Stress de } \\
\text { Lipp e ao Check List of Interpersonal } \\
\text { Transactions - II (CLOIT-II) }\end{array}$ & $\begin{array}{l}\text { Estado no Sudeste } \\
\text { brasileiro }\end{array}$ & $\begin{array}{l}\text { As posições interpessoais que mais fortemente se } \\
\text { relacionaram com estresse foram: Desconfiança, } \\
\text { Frieza Afetiva e Inibição. }\end{array}$ & $\begin{array}{l}\text { Padrões de interação interpessoais baseados } \\
\text { em características de hostilidade, como } \\
\text { queixar-se das situações, das tarefas que tem } \\
\text { para realizar e das pessoas com as quais } \\
\text { interage, estão ligadas a indicadores de } \\
\text { estresse, presentes há mais tempo. }\end{array}$ \\
\hline Tavares et al., 2017 & $\begin{array}{c}\text { 134 policiais } \\
\text { militares do } \\
\text { Batalhão de } \\
\text { Operações Especiais }\end{array}$ & $\begin{array}{l}\text { Estudo transversal e analítico. } \\
\text { Escala do Modelo Desequilíbrio } \\
\text { Esforço-Recompensa (DER) }\end{array}$ & Rio Grande do Sul & $\begin{array}{l}\text { Idade, PA diastólica, peso, circunferência } \\
\text { abdominal e circunferência do quadril, } \\
\text { relacionaram-se negativamente com os escores } \\
\text { de cortisol ao acordar. } \\
\text { O cortisol noite relacionou-se negativamente } \\
\text { com a circunferência abdominal e apresentou } \\
\text { maiores escores para os policiais não tabagistas e } \\
\text { sem relatos de problemas de saúde. }\end{array}$ & $\begin{array}{l}\text { A carga horária semanal de trabalho se } \\
\text { relacionou negativamente com o cortisol ao } \\
\text { acordar, ou seja, quanto maior a carga horária, } \\
\text { maior sobrecarga, mais frequente exposição a } \\
\text { estressores no trabalho, menor a resposta do } \\
\text { cortisol ao despertar. } \\
\text { A variação do cortisol salivar foi influenciada } \\
\text { por variáveis individuais, laborais e } \\
\text { psicossociais }\end{array}$ \\
\hline
\end{tabular}

Fonte: Autores. 
Em relação ao local das pesquisas, constatou-se que os estudos foram realizados em Natal (Rio Grande do Norte) (Costa et al., 2007); São Luís (Maranhão) (Carvalho et al., 2008), Rio de Janeiro (Bezerra, Minayo \& Constantino, 2013), Rio Grande do Sul (Tavares et al., 2017) e um não especificou a cidade, informando apenas que havia sido realizado na região do Sudeste Brasileiro (Couto, Vandenberghe \& Brito, 2012).

Analisando qualitativamente os artigos selecionados identificou-se no estudo realizado em São Luís, Maranhão que 26,7\% dos policiais da administração e 10,6\% do grupo com atividade operacional apresentaram estresse. A prevalência de bruxismo nesse estudo foi de 33,3\%. A prevalência de estresse em indivíduos com bruxismo foi significativamente maior em relação aos indivíduos sem bruxismo ( $p=0,003)$ (Carvalho et al., 2008).

No estudo realizado em Natal, Rio Grande do Norte, identificou-se que 125 (47,4\%) policiais apresentaram estresse. Destes, 3,4\% encontravam-se na fase de alerta, 39,8\% na fase de resistência, 3,8\% na fase de quase-exaustão e $0,4 \%$ na fase de exaustão. Quanto aos sintomas de estresse identificados tem-se 76,0\% policiais com sintomas psicológicos e 24,0\% com sintomas físicos (Costa et al., 2007).

Estudo realizado no Rio Grande do Sul identificou que a carga horária semanal de trabalho se relacionou negativamente com o cortisol ao acordar. Foi percebido que quanto maior a carga horária, maior a sobrecarga de trabalho, e mais frequente era a exposição a estressores no trabalho, menor era a resposta do cortisol ao despertar. Os autores alegam que isso pode sugerir que a baixa produção de cortisol ao despertar pode ser decorrência da Síndrome Metabólica e/ou de estresse (Tavares et al., 2017). No mesmo estudo identificou-se que o cortisol noite relacionou-se negativamente com a circunferência abdominal e apresentou maiores escores para os policiais não tabagistas e sem relatos de problemas de saúde. Segundo os autores isso pode ser devido ao fato de que os policiais com menor circunferência abdominal, que não fumavam e não tinham problemas de saúde seriam possivelmente mais ativos e comprometidos com o trabalho, encontrando dificuldades para se "desligar" do trabalho à noite.

Alguns fatores foram elencados como geradores de estresse. Dentre eles estão os padrões de interação interpessoal baseados em características de hostilidade, como queixar-se das situações, das tarefas que tem para realizar e das pessoas com as quais interage. Isso contribui com posturas profissionais de desconfiança, frieza afetiva e inibição (Couto, Vandenberghe \& Brito, 2012).

Outros fatores geradores de estresse estão relacionados aos problemas com a hierarquia, grande demanda de trabalho e preconceito de gênero (Almeida et al., 2020). Estudo realizado na cidade de Natal, Rio Grande do Norte identificou uma associação positiva entre o sexo feminino e estresse $(p=0,0337)$ (Costa et al., 2007). Percebe-se ainda, que o sofrimento psíquico aparece mais fortemente entre as mulheres oficiais com cargos de chefia, e as atividades operacionais são percebidas como mais estressantes pelo risco que oferecem (Bezerra, Minayo \& Constantino, 2013).

Um dos estudos sugeriu o exercício físico como estratégia eficaz para prevenir as consequências do estresse (Tavares et al., 2017). Essa revisão sistemática identificou que a hipótese sugerida no início do estudo era parcialmente verdadeira. O esforço físico não foi citado como fator estressante para os policiais militares nos artigos analisados, mas identificou-se que a interação interpessoal baseados em características de hostilidade (Couto, Vandenberghe \& Brito, 2012); problemas com a hierarquia, grande demanda de trabalho e preconceito de gênero (Bezerra, Minayo \& Constantino, 2013) eram fatores geradores de estresse.

A presença feminina nos batalhões consegue evidenciar um princípio de militarismo, onde prevalece não apenas a força física atribuída aos homens, mas também atributos individuais como: coragem e dedicação e essas são competências e habilidades que podem ser encontradas na mulher (Lara et al., 2017). A presença da mulher no mercado de trabalho se expandiu, devido a sua maior escolaridade e capacitação, fazendo com que a Polícia Militar visualizasse na figura da mulher a 
possibilidade de conferir à instituição uma aparência mais humana e democrática, diferente da imagem que tinha ligada diretamente ao período ditatorial (Souza, 2014).

Estudo que abordou a questão do gênero feminino na polícia militar do Rio de Janeiro identificou que mesmo dentro de uma instituição tão rígida como a Polícia Militar é possível encontrar espaços de negociação, que por sua vez apontem novos caminhos (Araújo, 2017).

Os artigos analisados nesta revisão permitem identificar que a presença de estresse em policiais militares é frequente. As frequências de casos de estresse variam entre os estudos, mas em todos foram elevadas. Identificou-se $26,7 \%$ dos policiais da administração e 10,6\% do grupo com atividade operacional apresentaram estresse (Carvalho et al., 2008); 125 (47,4\%) policiais apresentaram estresse, estando a maioria $(39,8 \%)$ na fase de resistência, e apresentando sintomas psicológicos (76,0\%) e sintomas físicos (24,0\%) (Costa et al., 2007). O estresse, seja ele de natureza biológica, emocional ou social, é composto por um conjunto de reações psicológicas que se forem exacerbadas com intensidade ou duração podem levar a um desequilíbrio no organismo. Os fatores ambientais nas relações interpessoais e a desorganização das tarefas de trabalho podem atuar também como estressor do organismo (Miranda \& Guimarães, 2016).

Um dos artigos selecionados alega que a baixa produção de cortisol ao despertar identificada em policiais militares pode ser decorrente da Síndrome Metabólica e/ou de estresse; e o cortisol alto a noite pode ser devido a dificuldade deles, em se "desligar" do trabalho à noite (Tavares et al., 2017). O trabalho policial leva o profissional a uma obrigatoriedade quanto à perfeita execução de suas atividades, que refletem numa exigência social em qualquer situação e sob quaisquer circunstâncias. Isso significa dizer que em muitas situações, o policial fica exposto a circunstâncias exaustivas e tensas, o que poderá desencadear o surgimento dos sintomas do estresse (Cordeiro et al., 2019). Como então, esse policial pode estar preparado para lidar com as situações do trabalho, se estes se encontram com sintomas de estresse? Estudo mostra que a formulação de atividades terapêuticas que visem à diminuição do estresse em policiais militares pode contribuir com o aumento de seu desempenho profissional, além de promover a melhoria na qualidade de vida dos mesmos (Neves et al., 2016).

Diante do elevado nível de estresse identificado no atual estudo percebe-se que é necessário formular tratamentos ou ações preventivas, como por exemplo, um acompanhamento mais direto aos policiais militares, considerando quais são os fatores geradores de estresse e como reduzi-los ou eliminá-los (Almeida et al., 2018). Outra contribuição das ações preventivas do estresse deve estar relacionada com as questões de gênero, tendo em vista que a organização e o gerenciamento no âmbito militar continuam sob a ótica masculina (Ribeiro \& Garcia, 2015). Para reduzir as vulnerabilidades e os riscos à saúde relacionados ao ambiente e ao processo de trabalho dos policiais, segundo a Política Nacional de Promoção da Saúde, faz-se necessário identificar os fatores associados ao comprometimento da qualidade de vida desses profissionais, os quais poderão subsidiar decisões e intervenções que promovam saúde e qualidade de vida (Malta et al., 2014).

O presente estudo identifica ainda a existência de poucos estudos sobre o estresse em policiais militares, reforçando a necessidade de mais estudos que contribuam com a identificação de outros fatores geradores de estresse e elaboração de medidas de prevenção do mesmo. Foram identificados dois estudos realizados na região nordeste, dois na região sudeste e um na região sul do Brasil. Isso reforça a necessidade de se estudar as demais regiões do Brasil, principalmente pela diversidade existente entre elas. Por se tratar de uma instituição com normas rígidas, a realização de estudos que demonstram as fragilidades dos policiais pode ser um dos motivos dessa baixa produção de estudos voltados ao tema proposto.

A falta de equilíbrio emocional pode levar os profissionais a adotarem atitudes irracionais durante crises e situações caóticas, que podem acarretar a falta de eficácia no desempenho do exercício profissional e expor os policiais e a população ao risco de dano e morte (Souza Filho et al., 2015). Diante disso, percebe-se que é necessário avançar na compreensão dos aspectos ambientais, psicológicos, sociais e físicos que envolvem a qualidade de vida dos policiais militares e implementar ações de promoção da saúde que melhorem as condições de vida, de saúde e de trabalho desses profissionais (Oliveira \& 
Quemelo, 2014). Isso permitirá o atendimento à Constituição Federal brasileira, onde a saúde é um direito fundamental expresso no artigo 6, como um direito social (BRASIL, 1988).

Algumas formas de minimizar os efeitos do estresse são: uma alimentação equilibrada (para repor os nutrientes que são gastos nos momentos mais estressantes), proporcionar momentos de relaxamento (com exercícios de respiração profunda, relaxamento muscular, ouvir músicas, assistir filmes e ler), fazer exercícios físicos regularmente e ter um acompanhamento psicológico de forma que gere uma qualidade de vida (Dantas et al., 2010).

O trabalho da polícia, mesmo sendo pacífica e em países desenvolvidos, é um trabalho difícil e estressante, com altos níveis de risco às exposições, a violência e aos horários de trabalho exaustivo. Esses trabalhadores têm um maior risco para transtornos mentais, como depressão, ansiedade, transtorno de estresse pós-traumático e suicídio, com maior prevalência que a população em geral (Gadelha et al., 2012). Isso reforça a importância de estudos com esse grupo de profissionais.

Pesquisas como esta, contribuem com reflexões sobre como conduzir o estresse entre os policiais militares. Couto et al. (2012) mostram que medidas de promoção da saúde, no sentido de auxiliar no controle adequado ao estresse visam melhorar a qualidade de vida, evitando sofrimento desnecessário. Além disso, podem interromper o ciclo do estresse e evitar a suscetibilidade dos policiais militares às doenças.

\section{Conclusão}

A interação interpessoal baseada em características de hostilidade, problemas com a hierarquia, grande demanda de trabalho e preconceito de gênero foram identificados como fatores geradores de estresse entre policiais militares. Além disso, a presença de estresse em policiais militares é frequente e traz comprometimentos psicológicos e físicos. Nesse contexto, a elaboração de ações de promoção de saúde e prevenção dos transtornos mentais dos policiais é necessária visando melhorar as condições laborais geradoras de estresse. O policial militar é um cidadão, portanto, deve ter direito a cuidados que vão da prevenção ao tratamento, com foco na melhoria da sua qualidade de vida.

Esta revisão apresenta limitações do estudo, pois não permite a generalização dos resultados para as demais regiões do Brasil. Assim, é importante o desenvolvimento de estudos capazes de identificar ao longo da carreira militar, os fatores geradores de estresse em policiais. Sugere-se ainda que sejam adotadas práticas de formação humanísticas e de autocontrole emocional, nos cursos preparatórios de novos policiais militares.

\section{Referências}

Almeida, D. M., Lopes, L. F. D., Costa, V. M. F., \& dos Santos, R. D. C. T. (2018). Policiais militares do estado do RS: relação entre satisfação no trabalho e estresse ocupacional. Administração Pública e Gestão Social, 10(1), 55-65.

Almeida, D. D., Lopes, L. F. D., Costa, V. M. F., Correa, J. S \& Menegazzi, R. B. (2020). Estresse Ocupacional e Relações de Gênero Entre Policiais Militares do Rio Grande do Sul. Revista FSA, 17, (11), 305-323.

Araujo, T. S. (2017). Mulheres em fardas policiais militares no Rio de Janeiro. Revista brasileira segurança pública, 11, (1), 74-96.

Bassalo, F. S., Ramos, M. F. H., Almeida,S. S. \& Silva, E. P. (2020). Autoeficácia e o Desempenho de soldados da Polícia Militar. Research, Society and Development, v9, (8), e701986485.

Bezerra, C. D. M., Minayo, M. C. D. S. \& Constantino, P. (2013). Estresse ocupacional em mulheres policiais. Ciência \& Saúde Coletiva, $18,657-666$.

Brasil. Constituição (1988). Constituição da República Federativa do Brasil. Brasília, DF: Senado Federal: Centro Gráfico, 1988.

Carvalho, S. C. A.; Carvalho, A. L. A., Lucena, S. C., Coelho, J. P. S., \& Araújo, T. P. B. (2008). Associação entre bruxismo e estresse em policiais militares. Revista Odonto Ciência, 23(2), 125-129.

Cordeiro, P., Batista, E. C., \& Oliveira, M. L. M. C. (2019). Avaliação do nível de estresse da polícia civil do interior do estado de Rondônia. Revista Interdisciplinar Encontro das Ciências, Icó, 2(1), 431-442.

Costa, M., Accioly Júnior, H. A., Oliveira, J., \& Maia, E. (2007). Estresse: diagnóstico dos policiais militares em uma cidade brasileira. Revista Panamericana de Salud Pública, 21, 217-222. 
Couto, G., Vandenberghe, L., \& Brito, E. D. A. G. (2012). Interações interpessoais e estresse entre policiais militares: um estudo correlacional. Arquivos Brasileiros de Psicologia, 64(2), 47-63.

Couto, G., Brito, E. D. A. G., Vasconcelos-Silva, A., \& Lucchese, R. (2017). Saúde mental do policial militar: Relações interpessoais e estresse no exercício profissional. Psicologia Argumento, 30(68), 185-194.

Dantas, M. A., Brito, D. V. C., Rodrigues, P. B., \& Maciente, T. S. (2010). Avaliação de estresse em policiais militares. Psicologia: teoria e prática, 12(3), 6677 .

Dorileo, A.W., \& Souza F. C. (2017) Qualidade de vida no trabalho: um estudo entre policiais militares do $12^{\circ}$ batalhão de Polícia Militar. Homens do mato Revista científica de pesquisa em segurança pública. 17 (3) 161-190.

Farnè, M. (2003). O estresse: às vezes é positivo, às vezes é negativo, mas pode ser transformado em um aliado. Paulinas.

Gadelha, A. L. Sarin, L. M. Leite, S. Ruy, N., Grinberg, L. P., \& Del Porto, J. A. (2012). Assessment of mood disorders prevalence in Brazilian military population. In Bipolar Disorders, 14, 74-74.

Lara, L. F., de Campos, E. A. R., Stefano, S. R., \& de Andrade, S. M. (2017). Relações de gênero na polícia militar: narrativas de mulheres policiais. Holos, 4, 56-77.

Lipp, M. E. N. (2005). Manual do inventário de sintomas de stress para adultos de Lipp (ISSL). Casa do Psicólogo.

Malta, D, Silva M, Albuquerque, G., Amorim R, Rodrigues, G., Silva T, et al. (2014). Política Nacional de Promoção da Saúde, descrição da implementação do eixo atividade física e práticas corporais, 2006 a 2014. Rev Bras Ativ Fís Saúde. 19: 286-99.

Minayo, M. C. D. S., Assis, S. G. D., \& Oliveira, R. V. C. D. (2011). Impacto das atividades profissionais na saúde física e mental dos policiais civis e militares do Rio de Janeiro (RJ, Brasil). Ciência \& Saúde Coletiva, 16, 2199-2209.

Minayo, M.C.S; \& Souza, E.R. (2003). Missão investigar: entre o ideal e a realidade de ser policial. Garamond.

Miranda, D. \& Guimarães, T. (2016). O Suicídio Policial: O que sabemos? DILEMAS Rev Estud Conflito e Control Soc, 9:13-34.

Neves, L., Oliveira, M. L. M. C., Ferreira, D. F., \& Batista, E. C. (2016). Sintomatologia de estresse em policiais militares numa cidade do interior de Rondônia. Revista Interdisciplinar Pensamento Científico, 2(1), 191-204.

Oliveira, K. L. D., \& Santos, L. M. D. (2010). Percepção da saúde mental em policiais militares da força tática e de rua. Sociologias, 12, 224-250.

Oliveira, L. C. N., \& Quemelo, P. R. V. (2014). Qualidade de vida de policiais militares. Arq Ciên Saúde, 21, 3, $72-75$.

Patis, K. (1987). Stress e isolamento social versus stress e atividade de resgate ao público: nota prévia. In Anais do Congresso da Associação Nacional de Medicina do Trabalho, 5, 785-789.

Ribeiro, A. N., \& Garcia, F. C. (2015). Relações de Poder e Gênero no Alto Comando da Polícia Militar de Minas Gerais: Uma Análise da Percepção das Mulheres Policiais. Teoria e Prática em Administração, 5(1), 53-79.

Silva, M. B. D., \& Vieira, S. B. (2008). O processo de trabalho do militar estadual e a saúde mental. Saúde e sociedade, 17, 161-170.

Silva, R., Schlichting, A. M., Schlichting, J. P., Gutierres Filho, P. J., Adami, F., \& Silva, A. (2012). Aspetos relacionados à qualidade de vida e atividade física de policiais militares de Santa Catarina-Brasil. Motricidade, 8(3), 81-89.

Souza, M. S. (2014). "Sou policial, mas sou mulher": gênero e representações sociais na Polícia Militar de São Paulo. Tese (doutorado). Universidade Estadual de Campinas, Instituto de Filosofia e Ciências Humanas. Campinas, SP: 441p.

Souza Filho, M. J. D., Noce, F., Andrade, A. G. P. D., Calixto, R. D. M., Albuquerque, M. R., \& Costa, V. T. (2015). Avaliação da qualidade de vida de policiais militares. R Bras Ci e Mov, 23(4), 159-69.

Souza, E. R. D., \& Minayo, M. C. D. S. (2005). Policial, risco como profissão: morbimortalidade vinculada ao trabalho. Ciência \& Saúde Coletiva, 10, 917928.

Tavares, J. P., Lautert, L., Magnago, T. S. B. D. S., Consiglio, A. R., \& Pai, D. D. (2017). Relationship between psychosocial stress dimensions and salivary cortisol in military police officers1. Revista latino-americana de enfermagem, 25.

Liberati, A., Altman, D. G., Tetzlaff, J., Mulrow, C., Gøtzsche, P. C., Ioannidis, J. P., \& Moher, D. (2009). The PRISMA statement for reporting systematic reviews and meta-analyses of studies that evaluate health care interventions: explanation and elaboration. Journal of clinical epidemiology, 62(10), e1-e34.

Shamseer, L., Moher, D., Clarke, M., Ghersi, D., Liberati, A., Petticrew, M., \& Stewart, L. A. (2015). Preferred reporting items for systematic review and meta-analysis protocols (PRISMA-P) 2015 statement. Systematic reviews, 4(1), 1-9.

Wright, B., Marshall, D., Adamson, J., Ainsworth, H., Ali, S., Allgar, V., \& Williams, C. (2016). Social Stories ${ }^{\mathrm{TM}}$ to alleviate challenging behaviour and social difficulties exhibited by children with autism spectrum disorder in mainstream schools: design of a manualised training toolkit and feasibility study for a cluster randomised controlled trial with nested qualitative and cost-effectiveness components. Health Technology Assessment 20(6), 1-258. 